ALEA, Lat. Am. J. Probab. Math. Stat. 14, 153-171 (2017)

DOI: 10.30757/ALEA.v14-09

\title{
Bounds for the asymptotic normality of the maximum likelihood estimator using the Delta method
}

\author{
Andreas Anastasiou and Christophe Ley \\ London School of Economics, Department of Statistics \\ Columbia House, Houghton Street, \\ WC2A 2AE \\ London, United Kingdom. \\ E-mail address: a.anastasiou@lse.ac.uk
}

Ghent University, Department of Applied Mathematics, Computer Science and Statistics Krijgslaan 281, S9, 9000

Gent, Belgium.

E-mail address: christophe.ley@ugent.be

\begin{abstract}
The asymptotic normality of the Maximum Likelihood Estimator (MLE) is a cornerstone of statistical theory. In the present paper, we provide sharp explicit upper bounds on Zolotarev-type distances between the exact, unknown distribution of the MLE and its limiting normal distribution. Our approach to this fundamental issue is based on a sound combination of the Delta method, Stein's method, Taylor expansions and conditional expectations, for the classical situations where the MLE can be expressed as a function of a sum of independent and identically distributed terms. This result is tailored for the broad class of one-parameter exponential family distributions.
\end{abstract}

\section{Introduction}

The asymptotic normality of maximum likelihood estimators (MLEs) is one of the best-known and most fundamental results in mathematical statistics. Under

Received by the editors April 4th, 2016; accepted February 2nd, 2017.

2010 Mathematics Subject Classification. 62F12, 62E17.

Key words and phrases. Delta method, Maximum likelihood estimator, Normal approximation, Stein's method.

A great part of this work has been completed while Andreas Anastasiou was working at the University of Oxford. Andreas Anastasiou was supported by a Teaching Assistantship Bursary from the Department of Statistics, University of Oxford, and EPSRC grant EP/K503113/1. Christophe Ley thanks the Fonds National de la Recherche Scientifique, Communauté Française de Belgique, for financial support via a Mandat de Chargé de Recherche FNRS.. 
certain regularity conditions (given later in this section), we have the following classical theorem, first discussed in Fisher (1925).

Theorem 1.1 (Asymptotic Normality of the MLE). Let $X_{1}, \ldots, X_{n}$ be i.i.d. random variables with probability density (or mass) function $f\left(x_{i} \mid \theta\right)$, where $\theta$ is a scalar parameter. Its true value is denoted by $\theta_{0}$. Assume that the MLE exists and it is unique and conditions (R1)-(R4), see below, are satisfied. Then

$$
\sqrt{n i\left(\theta_{0}\right)}\left(\hat{\theta}_{n}(\boldsymbol{X})-\theta_{0}\right) \underset{n \rightarrow \infty}{\stackrel{\mathrm{d}}{\longrightarrow}} \mathcal{N}(0,1)
$$

where $i\left(\theta_{0}\right)$ is the expected Fisher information quantity and $\stackrel{\mathrm{d}}{\rightarrow}$ means convergence in distribution.

The aim of the present paper is to complement this qualitative result with a quantitative statement, in other words, to find the best possible approximation for the distance, at finite sample size $n$, between the distribution of $\sqrt{n i\left(\theta_{0}\right)}\left(\hat{\theta}_{n}(\boldsymbol{X})-\theta_{0}\right)$ on the one hand and $\mathcal{N}(0,1)$ on the other hand. In mathematical terms, for $Z \sim \mathcal{N}(0,1)$, we are interested in the quantity

$$
\begin{aligned}
& d_{H}\left(\sqrt{n i\left(\theta_{0}\right)}\left(\hat{\theta}_{n}(\boldsymbol{X})-\theta_{0}\right), Z\right) \\
& =\sup _{h \in H}\left|\mathrm{E}\left[h\left(\sqrt{n i\left(\theta_{0}\right)}\left(\hat{\theta}_{n}(\boldsymbol{X})-\theta_{0}\right)\right)\right]-\mathrm{E}[h(Z)]\right|
\end{aligned}
$$

with

$$
H=\{h: \mathbb{R} \rightarrow \mathbb{R}, \text { absolutely continuous and bounded }\} .
$$

Distances of this type are called Zolotarev-type distances. Our focus will lie on the classical situations where the MLE can be expressed as a function of a sum of independent and identically distributed terms.

Consider an i.i.d. sample of observations $\boldsymbol{X}=\left(X_{1}, \ldots, X_{n}\right)$. Writing $\hat{\theta}_{n}(\boldsymbol{X})$ the MLE of the scalar parameter of interest $\theta \in \Theta \subseteq \mathbb{R}$, we are interested in settings where there exists a one-to-one twice differentiable mapping $q: \Theta \rightarrow \mathbb{R}$ with $q^{\prime}(\theta) \neq 0 \forall \theta \in \Theta$ such that

$$
q\left(\hat{\theta}_{n}(\boldsymbol{X})\right)=\frac{1}{n} \sum_{i=1}^{n} g\left(X_{i}\right)
$$

for some $g: \mathbb{R} \rightarrow \mathbb{R}$. Situations of this kind are all but rare; with $f(x \mid \theta)$ the probability density (or mass) function, classical examples include

- the normal distribution with density $f\left(x \mid \mu, \sigma^{2}\right)=\frac{1}{\sigma \sqrt{2 \pi}} \exp \left(-\frac{1}{2 \sigma^{2}}(x-\mu)^{2}\right)$, $x \in \mathbb{R}$, for which $\mu \in \mathbb{R}$ is our unknown parameter, whereas $\sigma>0$ is considered to be known. The MLE for $\theta=\mu$ is

$$
\hat{\theta}_{n}(\boldsymbol{X})=\frac{1}{n} \sum_{i=1}^{n} X_{i}
$$

- the normal distribution, where now the mean $\mu$ is known and $\theta=\sigma^{2}$ represents the unknown parameter, with

$$
\hat{\theta}_{n}(\boldsymbol{X})=\frac{1}{n} \sum_{i=1}^{n}\left(X_{i}-\mu\right)^{2} ;
$$


- the Weibull distribution with density $f(x \mid \alpha, \sigma)=\frac{\alpha}{\sigma}\left(\frac{x}{\sigma}\right)^{\alpha-1} \exp \left(-\left(\frac{x}{\sigma}\right)^{\alpha}\right)$, $x \geq 0$, where $\sigma$ is the unknown scale parameter and $\alpha>0$ is fixed. The MLE for $\theta=\sigma$ is defined through

$$
\left(\hat{\theta}_{n}(\boldsymbol{X})\right)^{\alpha}=\frac{1}{n} \sum_{i=1}^{n} X_{i}^{\alpha}
$$

- the Laplace scale model with density $f(x \mid \sigma)=\frac{1}{2 \sigma} \exp (-|x| / \sigma), \theta=\sigma>0$, over $\mathbb{R}$, for which

$$
\hat{\theta}_{n}(\boldsymbol{X})=\frac{1}{n} \sum_{i=1}^{n}\left|X_{i}\right|
$$

The broad one-parameter exponential families do satisfy condition (1.3); actually, it is possible to show that MLEs of the form (1.3) are characteristic of these families, see Proposition 3.1 for details. Hence, our results do apply to most of the wellknown distributions.

We now present in detail the notation used throughout the paper. We write $\mathrm{E}_{\theta}$ [] the expectation under the specific value $\theta$ of the parameter. In line with the notation used above, the joint density or probability mass function of $\boldsymbol{X}$ is written $f(\boldsymbol{x} \mid \theta)$. The true, unknown value of the parameter is $\theta_{0}$ and $\Theta$ denotes the parameter space. For $X_{i}=x_{i}$ some observed values, the likelihood function is denoted by $L(\theta ; \boldsymbol{x})=f(\boldsymbol{x} \mid \theta)$ and we denote its natural logarithm, called the log-likelihood function, by $l(\theta ; \boldsymbol{x})$. The derivatives of the log-likelihood function with respect to $\theta$ are $l^{\prime}(\theta ; \boldsymbol{x}), l^{\prime \prime}(\theta ; \boldsymbol{x}), \ldots, l^{(j)}(\theta ; \boldsymbol{x})$, for $j$ any integer greater than 2 , and $i(\theta)$ denotes the expected Fisher information number for one random variable. Whenever the MLE exists and is also unique, we will write it as before under the form $\hat{\theta}_{n}(\boldsymbol{X})$. For $\Theta$ being an open interval, we use the results in Mäkeläinen et al. (1981) to secure the existence and uniqueness of the MLE. Thus, it suffices to assume that:

(A1) The log-likelihood function $l(\theta ; \boldsymbol{x})$ is a twice continuously differentiable function with respect to $\theta$ and the parameter varies in an open interval $(a, b)$, where $a, b \in \mathbb{R} \cup\{-\infty, \infty\}$ and $a<b$;

(A2) $\lim _{\theta \rightarrow a b} l(\theta ; \boldsymbol{x})=-\infty$;

(A3) $l^{\prime \prime}(\theta ; \boldsymbol{x})<0$ at every point $\theta \in(a, b)$ for which $l^{\prime}(\theta ; \boldsymbol{x})=0$.

Note that we tacitly assume those conditions in Theorem 1.1 when requiring existence and uniqueness of the MLE. Asymptotic normality further requires the following sufficient regularity conditions:

(R1) the parameter is identifiable, which means that if $\theta \neq \theta^{\prime}$, then $\exists x: f(x \mid \theta) \neq$ $f\left(x \mid \theta^{\prime}\right)$

(R2) the density $f(x \mid \theta)$ is three times differentiable with respect to $\theta$, the third derivative is continuous in $\theta$ and $\int f(x \mid \theta) \mathrm{d} x$ can be differentiated three times under the integral sign;

(R3) for any $\theta_{0} \in \Theta$ and for $\mathbb{X}$ denoting the support of $f(x \mid \theta)$, there exists a positive number $\epsilon$ and a function $M(x)$ (both of which may depend on $\theta_{0}$ ) such that

$$
\left|\frac{\mathrm{d}^{3}}{\mathrm{~d} \theta^{3}} \log f(x \mid \theta)\right| \leq M(x) \quad \forall x \in \mathbb{X}, \quad \theta_{0}-\epsilon<\theta<\theta_{0}+\epsilon,
$$

with $\mathrm{E}_{\theta_{0}}[M(X)]<\infty$;

(R4) $i(\theta)>0, \forall \theta \in \Theta$. 
These conditions, in particular (R2), ensure that, provided the respective expressions exist, $\mathrm{E}_{\theta}\left[l^{\prime}(\theta ; \boldsymbol{X})\right]=0$ and $\operatorname{Var}_{\theta}\left[l^{\prime}(\theta ; \boldsymbol{X})\right]=n i(\theta)$. These conditions form the basis of Theorem 1.1 above; see page 472 of Casella and Berger (1990) for a basic sketch of the proof.

The first paper to address the problem of assessing the accuracy of the normal approximation for the MLE is Anastasiou and Reinert (2017). After deriving general bounds on Zolotarev-type distances, they use the bounded Wasserstein distance $d_{b W}$, which is also known as Fortet-Mourier distance (see, e.g., Nourdin and Peccati, $2012)$ and is linked to the Kolmogorov distance ( $H$ is the class of indicator functions of half-spaces) via $d_{K}(\cdot, \cdot) \leq 2 \sqrt{d_{b W}(\cdot, \cdot)}$. We state in Theorem 2.4 of Section 2 the bound obtained in that paper. For the broad class of distributions satisfying (1.3), our bound is better than, or at least as good as, the Anastasiou and Reinert (2017) bound (hereafter referred to as AR-bound) both in terms of sharpness and simplicity. The tools we use to reach this result are the Delta method, Stein's method for normal approximation, Taylor expansions and conditional expectations.

The paper is organised as follows. Our new upper bound is described, proved and compared to the AR-bound in Section 2, and practical statistical applications are exhibited. In Section 3 we then apply our results to the class of one-parameter exponential family distributions and treat some specific examples in detail.

\section{New bounds on the distance to the normal distribution for the MLE}

In order to obtain bounds on the aforementioned distance, we partly employ the following lemma. From now on, unless otherwise stated, $\|\cdot\|$ denotes the infinity norm $\|\cdot\|_{\infty}$. Also, for the sake of presentation we drop the subscript $\theta_{0}$ from the expectation and variance.

Lemma 2.1 (Reinert, 1998). Let $Y_{1}, \ldots, Y_{n}$ be independent random variables with $\mathrm{E}\left(Y_{i}\right)=0, \operatorname{Var}\left(Y_{i}\right)=\sigma^{2}>0$ and $\mathrm{E}\left[\left|Y_{i}\right|^{3}\right]<\infty$. Let $W=\frac{1}{\sqrt{n}} \sum_{i=1}^{n} Y_{i}$, with $\mathrm{E}(W)=0, \operatorname{Var}(W)=\sigma^{2}$ and let $K \sim \mathcal{N}\left(0, \sigma^{2}\right)$. Then for any function $h \in H$, with $H$ given in (1.2), one has

$$
|\mathrm{E}[h(W)]-\mathrm{E}[h(K)]| \leq \frac{\left\|h^{\prime}\right\|}{\sqrt{n}}\left(2+\frac{1}{\sigma^{3}} \mathrm{E}\left[\left|Y_{1}\right|^{3}\right]\right) .
$$

As we shall see below, our strategy consists in benefiting from the special form of $q\left(\hat{\theta}_{n}(\boldsymbol{X})\right)$, which is a sum of random variables and thus allows us to use the sharp bound of this lemma. It is precisely at this point that the Delta method comes into play: abusing notations and language, instead of comparing $\hat{\theta}_{n}(\boldsymbol{X})$ to $Z \sim \mathcal{N}(0,1)$ we rather compare $q\left(\hat{\theta}_{n}(\boldsymbol{X})\right)$ to $Z$, and then bound the distance between $\hat{\theta}_{n}(\boldsymbol{X})$ and $q\left(\hat{\theta}_{n}(\boldsymbol{X})\right)$. The outcome of this approach is the next theorem, the main result of the present paper.

Theorem 2.2. Let $X_{1}, \ldots, X_{n}$ be i.i.d. random variables with probability density (or mass) function $f\left(x_{i} \mid \theta\right)$ and let $Z \sim \mathcal{N}(0,1)$. Assume that (R1)-(R4) are satisfied. Furthermore, assume that the MLE exists and (1.3) is satisfied. In addition, the mapping $g: \mathbb{R} \rightarrow \mathbb{R}$ is such that $\mathrm{E}\left[\left|g\left(X_{1}\right)-q\left(\theta_{0}\right)\right|^{3}\right]<\infty$ for $\theta_{0} \in \Theta$ the true value of the parameter. Then, for any $h \in H$ as in (1.2), 
(1) if $q^{\prime \prime}(\cdot)$ is not uniformly bounded but for any $\theta_{0} \in \Theta$ there exists $0<\epsilon=$ $\epsilon\left(\theta_{0}\right)$ such that $\sup _{\theta:\left|\theta-\theta_{0}\right|<\epsilon}\left|q^{\prime \prime}(\theta)\right|<\infty$, we have

$$
\begin{aligned}
& \left|\mathrm{E}\left[h\left(\sqrt{n i\left(\theta_{0}\right)}\left(\hat{\theta}_{n}(\boldsymbol{X})-\theta_{0}\right)\right)\right]-\mathrm{E}[h(Z)]\right| \\
& \leq \frac{\left\|h^{\prime}\right\|}{\sqrt{n}}\left(2+\frac{\left[i\left(\theta_{0}\right)\right]^{\frac{3}{2}}}{\left|q^{\prime}\left(\theta_{0}\right)\right|^{3}} \mathrm{E}\left[\left|g\left(X_{1}\right)-q\left(\theta_{0}\right)\right|^{3}\right]\right) \\
& +\mathrm{E}\left[\left(\hat{\theta}_{n}(\boldsymbol{X})-\theta_{0}\right)^{2}\right]\left(2 \frac{\|h\|}{\epsilon^{2}} \mathbb{1}\{\exists \theta \in \Theta: q(\theta) \neq \theta\}+\frac{\left\|h^{\prime}\right\| \sqrt{n i\left(\theta_{0}\right)}}{2\left|q^{\prime}\left(\theta_{0}\right)\right|} \sup _{\theta:\left|\theta-\theta_{0}\right| \leq \epsilon}\left|q^{\prime \prime}(\theta)\right|\right) ;
\end{aligned}
$$

(2) if $q^{\prime \prime}(\cdot)$ is uniformly bounded, with $\left|q^{\prime \prime}(\theta)\right| \leq B^{*} \forall \theta \in \Theta$ for some $B^{*}>0$, we have

$$
\begin{aligned}
& \left|\mathrm{E}\left[h\left(\sqrt{n i\left(\theta_{0}\right)}\left(\hat{\theta}_{n}(\boldsymbol{X})-\theta_{0}\right)\right)\right]-\mathrm{E}[h(Z)]\right| \\
& \leq \frac{\left\|h^{\prime}\right\|}{\sqrt{n}}\left(2+\frac{\left[i\left(\theta_{0}\right)\right]^{\frac{3}{2}}}{\left|q^{\prime}\left(\theta_{0}\right)\right|^{3}} \mathrm{E}\left[\left|g\left(X_{1}\right)-q\left(\theta_{0}\right)\right|^{3}\right]\right) \\
& +\left\|h^{\prime}\right\| B^{*} \frac{\sqrt{n i\left(\theta_{0}\right)}}{2\left|q^{\prime}\left(\theta_{0}\right)\right|} \mathrm{E}\left[\left(\hat{\theta}_{n}(\boldsymbol{X})-\theta_{0}\right)^{2}\right] \mathbb{1}\{\exists \theta \in \Theta: q(\theta) \neq \theta\} .
\end{aligned}
$$

Proof: The asymptotic normality of the MLE is explicitly stated in Theorem 1.1. Applying the widely known Delta method to this result in combination with the requirement $q^{\prime}\left(\theta_{0}\right) \neq 0$ yields

$$
\frac{\sqrt{n i\left(\theta_{0}\right)}}{q^{\prime}\left(\theta_{0}\right)}\left(q\left(\hat{\theta}_{n}(\boldsymbol{X})\right)-q\left(\theta_{0}\right)\right) \underset{n \rightarrow \infty}{\stackrel{\mathrm{d}}{\longrightarrow}} \mathcal{N}(0,1)
$$

with $q\left(\hat{\theta}_{n}(\boldsymbol{X})\right)=\frac{1}{n} \sum_{i=1}^{n} g\left(X_{i}\right)$. Using the triangle inequality,

$$
\begin{aligned}
& \left|\mathrm{E}\left[h\left(\sqrt{n i\left(\theta_{0}\right)}\left(\hat{\theta}_{n}(\boldsymbol{X})-\theta_{0}\right)\right)\right]-\mathrm{E}[h(Z)]\right| \\
& \leq\left|\mathrm{E}\left[h\left(\frac{\sqrt{n i\left(\theta_{0}\right)}}{q^{\prime}\left(\theta_{0}\right)}\left(q\left(\hat{\theta}_{n}(\boldsymbol{X})\right)-q\left(\theta_{0}\right)\right)\right)\right]-\mathrm{E}[h(Z)]\right| \\
& +\left|\mathrm{E}\left[h\left(\sqrt{n i\left(\theta_{0}\right)}\left(\hat{\theta}_{n}(\boldsymbol{X})-\theta_{0}\right)\right)-h\left(\frac{\sqrt{n i\left(\theta_{0}\right)}}{q^{\prime}\left(\theta_{0}\right)}\left(q\left(\hat{\theta}_{n}(\boldsymbol{X})\right)-q\left(\theta_{0}\right)\right)\right)\right]\right| .
\end{aligned}
$$

We first obtain an upper bound for (2.4) using (indirectly) Stein's method via Lemma 2.1. Some simple rewriting yields

$$
\begin{aligned}
& \frac{\sqrt{n i\left(\theta_{0}\right)}}{q^{\prime}\left(\theta_{0}\right)}\left(q\left(\hat{\theta}_{n}(\boldsymbol{X})\right)-q\left(\theta_{0}\right)\right)=\frac{\sqrt{n i\left(\theta_{0}\right)}}{q^{\prime}\left(\theta_{0}\right)}\left(\frac{1}{n} \sum_{i=1}^{n} g\left(X_{i}\right)-q\left(\theta_{0}\right)\right) \\
& =\frac{1}{\sqrt{n}} \sum_{i=1}^{n}\left\{\frac{\sqrt{i\left(\theta_{0}\right)}}{q^{\prime}\left(\theta_{0}\right)}\left(g\left(X_{i}\right)-q\left(\theta_{0}\right)\right)\right\}=\frac{1}{\sqrt{n}} \sum_{i=1}^{n} Y_{i},
\end{aligned}
$$


where $Y_{i}=\frac{\sqrt{i\left(\theta_{0}\right)}}{q^{\prime}\left(\theta_{0}\right)}\left(g\left(X_{i}\right)-q\left(\theta_{0}\right)\right), i=1,2, \ldots, n$ and, obviously, the $Y_{i}$ 's are independent and identically distributed random variables. The Central Limit Theorem applied to $\frac{1}{\sqrt{n}} \sum_{i=1}^{n} Y_{i}$ implies that $\frac{1}{\sqrt{n}} \sum_{i=1}^{n}\left(Y_{i}-\mathrm{E}\left(Y_{1}\right)\right) \underset{n \rightarrow \infty}{\stackrel{\mathrm{d}}{\longrightarrow}} \mathcal{N}\left(0, \operatorname{Var}\left(Y_{1}\right)\right)$. From (2.3) we know however that $\frac{1}{\sqrt{n}} \sum_{i=1}^{n} Y_{i} \underset{n \rightarrow \infty}{\stackrel{\mathrm{d}}{\longrightarrow}} \mathcal{N}(0,1)$; comparing the two asymptotic results reveals that, necessarily (since two normal distributions can only be equal if their expectations and variances are the same), we have

$$
\mathrm{E}\left[g\left(X_{1}\right)\right]=q\left(\theta_{0}\right) \quad \text { and } \quad \operatorname{Var}\left[g\left(X_{1}\right)\right]=\frac{\left(q^{\prime}\left(\theta_{0}\right)\right)^{2}}{i\left(\theta_{0}\right)},
$$

where condition (R4) allows to divide by $i\left(\theta_{0}\right)$. Hence, as Lemma 2.1 requires, $\mathrm{E}\left[Y_{i}\right]=0, \operatorname{Var}\left[Y_{i}\right]=1$, and $\mathrm{E}\left[\left|Y_{i}\right|^{3}\right]<\infty$ thanks to the additional condition on $g$. Applying the result of the lemma we get

$$
\begin{aligned}
& \left|\mathrm{E}\left[h\left(\frac{\sqrt{n i\left(\theta_{0}\right)}}{q^{\prime}\left(\theta_{0}\right)}\left(q\left(\hat{\theta}_{n}(\boldsymbol{X})\right)-q\left(\theta_{0}\right)\right)\right)\right]-\mathrm{E}[h(Z)]\right| \\
& \leq \frac{\left\|h^{\prime}\right\|}{\sqrt{n}}\left(2+\frac{\left[i\left(\theta_{0}\right)\right]^{\frac{3}{2}}}{\left|q^{\prime}\left(\theta_{0}\right)\right|^{3}} \mathrm{E}\left[\left|g\left(X_{1}\right)-q\left(\theta_{0}\right)\right|^{3}\right]\right) .
\end{aligned}
$$

Now we are searching for an upper bound on (2.5). Since the case $q(\theta)=\theta \forall \theta \in \Theta$ is obvious, we from here on assume that $q(\theta) \neq \theta$. We denote

$$
\begin{aligned}
A & :=A\left(q, \theta_{0}, \boldsymbol{X}\right) \\
& :=h\left(\sqrt{n i\left(\theta_{0}\right)}\left(\hat{\theta}_{n}(\boldsymbol{X})-\theta_{0}\right)\right)-h\left(\frac{\sqrt{n i\left(\theta_{0}\right)}}{q^{\prime}\left(\theta_{0}\right)}\left(q\left(\hat{\theta}_{n}(\boldsymbol{X})\right)-q\left(\theta_{0}\right)\right)\right)
\end{aligned}
$$

and our scope is to find an upper bound for $|\mathrm{E}[A]|$.

Case 1: $\forall \theta \in \Theta$ there exists $0<\epsilon=\epsilon\left(\theta_{0}\right)$ such that $\sup _{\theta:\left|\theta-\theta_{0}\right|<\epsilon}\left|q^{\prime \prime}(\theta)\right|<\infty$. Then, using the law of total expectation related to conditioning on $\left|\hat{\theta}_{n}(\boldsymbol{X})-\theta_{0}\right| \geq \epsilon$ or $\left|\hat{\theta}_{n}(\boldsymbol{X})-\theta_{0}\right|<\epsilon$ yields

$$
\begin{aligned}
|\mathrm{E}[A]| \leq \mathrm{E}[|A|]= & \mathrm{E}\left[|A||| \hat{\theta}_{n}(\boldsymbol{X})-\theta_{0} \mid \geq \epsilon\right] \mathbb{P}\left(\left|\hat{\theta}_{n}(\boldsymbol{X})-\theta_{0}\right| \geq \epsilon\right) \\
& +\mathrm{E}\left[|A||| \hat{\theta}_{n}(\boldsymbol{X})-\theta_{0} \mid<\epsilon\right] \mathbb{P}\left(\left|\hat{\theta}_{n}(\boldsymbol{X})-\theta_{0}\right|<\epsilon\right) .
\end{aligned}
$$

Markov's inequality and the elementary results of $\mathbb{P}\left(\left|\hat{\theta}_{n}(\boldsymbol{X})-\theta_{0}\right|<\epsilon\right) \leq 1$ and $|A| \leq 2\|h\|$ further yield

$$
|\mathrm{E}(A)| \leq 2\|h\| \frac{\mathrm{E}\left[\left(\hat{\theta}_{n}(\boldsymbol{X})-\theta_{0}\right)^{2}\right]}{\epsilon^{2}}+\mathrm{E}\left[|A||| \hat{\theta}_{n}(\boldsymbol{X})-\theta_{0} \mid<\epsilon\right] .
$$

We now focus on the conditional expectation on the right-hand side of (2.7). A second-order Taylor expansion of $q\left(\hat{\theta}_{n}(\boldsymbol{x})\right)$ about $\theta_{0}$ gives

$$
q\left(\hat{\theta}_{n}(\boldsymbol{x})\right)=q\left(\theta_{0}\right)+\left(\hat{\theta}_{n}(\boldsymbol{x})-\theta_{0}\right) q^{\prime}\left(\theta_{0}\right)+\frac{1}{2}\left(\hat{\theta}_{n}(\boldsymbol{x})-\theta_{0}\right)^{2} q^{\prime \prime}\left(\theta^{*}\right),
$$


for $\theta^{*}$ between $\hat{\theta}_{n}(\boldsymbol{x})$ and $\theta_{0}$. Since $q^{\prime}(\theta) \neq 0 \forall \theta \in \Theta$ is assumed, we can multiply both sides in (2.8) with $\frac{\sqrt{n i\left(\theta_{0}\right)}}{q^{\prime}\left(\theta_{0}\right)}$. Rearranging the terms,

$$
\begin{aligned}
& \frac{\sqrt{n i\left(\theta_{0}\right)}\left(q\left(\hat{\theta}_{n}(\boldsymbol{x})\right)-q\left(\theta_{0}\right)\right)}{q^{\prime}\left(\theta_{0}\right)} \\
& =\sqrt{n i\left(\theta_{0}\right)}\left(\hat{\theta}_{n}(\boldsymbol{x})-\theta_{0}\right)+\frac{\sqrt{n i\left(\theta_{0}\right)}}{2 q^{\prime}\left(\theta_{0}\right)} q^{\prime \prime}\left(\theta^{*}\right)\left(\hat{\theta}_{n}(\boldsymbol{x})-\theta_{0}\right)^{2} .
\end{aligned}
$$

The above result along with another first-order Taylor expansion (recall that $\sqrt{n}\left(\hat{\theta}_{n}(\boldsymbol{X})-\theta_{0}\right)^{2}=o_{\mathrm{P}}(1)$ as $\left.n \rightarrow \infty\right)$ gives

$$
\begin{aligned}
& h\left(\sqrt{n i\left(\theta_{0}\right)}\left(\hat{\theta}_{n}(\boldsymbol{x})-\theta_{0}\right)\right)-h\left(\frac{\sqrt{n i\left(\theta_{0}\right)}}{q^{\prime}\left(\theta_{0}\right)}\left(q\left(\hat{\theta}_{n}(\boldsymbol{x})\right)-q\left(\theta_{0}\right)\right)\right) \\
& =-\frac{\sqrt{n i\left(\theta_{0}\right)}}{2 q^{\prime}\left(\theta_{0}\right)} q^{\prime \prime}\left(\theta^{*}\right)\left(\hat{\theta}_{n}(\boldsymbol{x})-\theta_{0}\right)^{2} h^{\prime}(t(\boldsymbol{x})),
\end{aligned}
$$

where $t(\boldsymbol{x})$ is between $\sqrt{n i\left(\theta_{0}\right)}\left(\hat{\theta}_{n}(\boldsymbol{x})-\theta_{0}\right)$ and $\frac{\sqrt{n i\left(\theta_{0}\right)}}{q^{\prime}\left(\theta_{0}\right)}\left(q\left(\hat{\theta}_{n}(\boldsymbol{x})\right)-q\left(\theta_{0}\right)\right)$. Equality (2.9) combined with Lemma 2.1 in Anastasiou and Reinert (2017) related to conditional expectations yield

$$
\begin{aligned}
& \mathrm{E}\left[|A||| \hat{\theta}_{n}(\boldsymbol{X})-\theta_{0} \mid<\epsilon\right] \\
& =\mathrm{E}\left[\left|-\frac{\sqrt{n i\left(\theta_{0}\right)}}{2 q^{\prime}\left(\theta_{0}\right)} q^{\prime \prime}\left(\theta^{*}\right)\left(\hat{\theta}_{n}(\boldsymbol{X})-\theta_{0}\right)^{2} h^{\prime}(t(\boldsymbol{X}))\right||| \hat{\theta}_{n}(\boldsymbol{X})-\theta_{0} \mid<\epsilon\right] \\
& \leq \frac{\left\|h^{\prime}\right\| \sqrt{n i\left(\theta_{0}\right)}}{2\left|q^{\prime}\left(\theta_{0}\right)\right|} \mathrm{E}\left[\left|q^{\prime \prime}\left(\theta^{*}\right)\right|\left(\hat{\theta}_{n}(\boldsymbol{X})-\theta_{0}\right)^{2}|| \hat{\theta}_{n}(\boldsymbol{X})-\theta_{0} \mid<\epsilon\right] \\
& \leq \frac{\left\|h^{\prime}\right\| \sqrt{n i\left(\theta_{0}\right)}}{2\left|q^{\prime}\left(\theta_{0}\right)\right|} \sup _{\theta:\left|\theta-\theta_{0}\right|<\epsilon}\left|q^{\prime \prime}(\theta)\right| \mathrm{E}\left[\left(\hat{\theta}_{n}(\boldsymbol{X})-\theta_{0}\right)^{2}|| \hat{\theta}_{n}(\boldsymbol{X})-\theta_{0} \mid<\epsilon\right] \\
& \leq \frac{\left\|h^{\prime}\right\| \sqrt{n i\left(\theta_{0}\right)}}{2\left|q^{\prime}\left(\theta_{0}\right)\right|} \sup _{\theta:\left|\theta-\theta_{0}\right|<\epsilon}\left|q^{\prime \prime}(\theta)\right| \mathrm{E}\left[\left(\hat{\theta}_{n}(\boldsymbol{X})-\theta_{0}\right)^{2}\right] .
\end{aligned}
$$

Combining the bounds in (2.6), (2.7) and (2.10) we get the result in (2.1).

Case 2: There exists $B^{*}>0$ such that $q^{\prime \prime}(\cdot)$ is uniformly bounded with $\left|q^{\prime \prime}(\theta)\right| \leq B^{*} \forall \theta \in \Theta$. In this case, we do not need to take conditional expectations. The result in (2.9) gives

$$
\begin{aligned}
& \mathrm{E}[|A|] \leq \frac{\left\|h^{\prime}\right\| \sqrt{n i\left(\theta_{0}\right)}}{2\left|q^{\prime}\left(\theta_{0}\right)\right|} \mathrm{E}\left[\left|q^{\prime \prime}\left(\theta^{*}\right)\right|\left(\hat{\theta}_{n}(\boldsymbol{X})-\theta_{0}\right)^{2}\right] \\
& \leq B^{*} \frac{\left\|h^{\prime}\right\| \sqrt{n i\left(\theta_{0}\right)}}{2\left|q^{\prime}\left(\theta_{0}\right)\right|} \mathrm{E}\left[\left(\hat{\theta}_{n}(\boldsymbol{X})-\theta_{0}\right)^{2}\right] .
\end{aligned}
$$

The above result and the bound in (2.6) give the bound in (2.2) for the case where $q^{\prime \prime}(\cdot)$ is uniformly bounded in $\theta$.

Remark 2.3. (1) The convergence of the terms (apart from the first one) is governed by the asymptotic behaviour of the mean squared error $\mathrm{E}\left[\left(\hat{\theta}_{n}(\boldsymbol{X})-\theta_{0}\right)^{2}\right]$, whose 
rate of convergence is $\mathcal{O}\left(\frac{1}{n}\right)$. This result is obtained using the decomposition

$$
\mathrm{E}\left[\left(\hat{\theta}_{n}(\boldsymbol{X})-\theta_{0}\right)^{2}\right]=\operatorname{Var}\left[\hat{\theta}_{n}(\boldsymbol{X})\right]+\operatorname{bias}^{2}\left[\hat{\theta}_{n}(\boldsymbol{X})\right]
$$

Under the standard asymptotics (from the regularity conditions) the MLE is asymptotically efficient, meaning that

$$
n \operatorname{Var}\left[\hat{\theta}_{n}(\boldsymbol{X})\right] \underset{n \rightarrow \infty}{\longrightarrow}\left[i\left(\theta_{0}\right)\right]^{-1}
$$

and hence the variance of the MLE is of order $\frac{1}{n}$. In addition, from Theorem 1.1 the bias of the MLE is of order $\frac{1}{\sqrt{n}}$; see also Cox and Snell (1968), where no explicit conditions are given. Combining these two results and using (2.12) shows that the mean squared error of the MLE is of order $\frac{1}{n}$.

(2) In the simplest possible situation where $\hat{\theta}_{n}(\boldsymbol{X})$ is already a sum of i.i.d. terms, $q(x)=x$ and hence our upper bound simplifies to

$$
\begin{aligned}
& \left|\mathrm{E}\left[h\left(\sqrt{n i\left(\theta_{0}\right)}\left(\hat{\theta}_{n}(\boldsymbol{X})-\theta_{0}\right)\right)\right]-\mathrm{E}[h(Z)]\right| \\
& \leq \frac{\left\|h^{\prime}\right\|}{\sqrt{n}}\left(2+\left[i\left(\theta_{0}\right)\right]^{\frac{3}{2}} \mathrm{E}\left[\left|g\left(X_{1}\right)-\theta_{0}\right|^{3}\right]\right),
\end{aligned}
$$

which is equivalent to Lemma 2.1.

(3) One may wonder whether asking (R1)-(R4) to hold is not a too strong assumption given that we assume the MLE to be of the special form (1.3). Indeed, one can easily get from the Central Limit Theorem that $\sqrt{n}\left(q(\hat{\theta}(\boldsymbol{X}))-\mathrm{E}\left[g\left(X_{1}\right)\right]\right) \underset{n \rightarrow \infty}{\stackrel{\mathrm{d}}{\longrightarrow}} \mathcal{N}\left(0, \operatorname{Var}\left[g\left(X_{1}\right)\right]\right)$ without needing the asymptotic normality of $\hat{\theta}(\boldsymbol{X})$ through (R1)-(R4). However, without this asymptotic normality of $\hat{\theta}(\boldsymbol{X})$ we cannot obtain (2.3), and without the latter explicit asymptotic result we cannot link $\mathrm{E}\left[g\left(X_{1}\right)\right]$ and $\operatorname{Var}\left[g\left(X_{1}\right)\right]$ to their corresponding $q$-based quantities. The sequel of the proof would boil down. This is the (perhaps not so obvious) reason why from the beginning of the proof we need the regularity conditions (R1)-(R4).

In order to appreciate the sharpness and simplicity of our new bounds, we compare our result to the AR-bound. For ease of presentation the comparison is concerned with the case where $q^{\prime \prime}(\cdot)$ is not uniformly bounded, but only bounded in a neightbourhood of $\theta_{0}$. Therefore, we employ the result in (2.1). Similar results hold when $q^{\prime \prime}(\cdot)$ is uniformly bounded. We now state the main result of Anastasiou and Reinert (2017).

Theorem 2.4 (Anastasiou and Reinert, 2017). Let $X_{1}, X_{2}, \ldots, X_{n}$ be i.i.d. random variables with density or frequency function $f\left(x_{i} \mid \theta\right)$ such that the regularity conditions (R1)-(R4) are satisfied and that the $M L E, \hat{\theta}_{n}(\boldsymbol{X})$, exists and it is unique. Assume that $\mathrm{E}\left[\left|\left(\frac{\mathrm{d}}{\mathrm{d} \theta} \log f\left(X_{1} \mid \theta\right)\right)_{\theta=\theta_{0}}\right|^{3}\right]<\infty$ and that $\mathrm{E}\left[\left(\hat{\theta}_{n}(\boldsymbol{X})-\theta_{0}\right)^{4}\right]<\infty$. Let $0<\epsilon=\epsilon\left(\theta_{0}\right)$ be such that $\left(\theta_{0}-\epsilon, \theta_{0}+\epsilon\right) \subset \Theta$ as in (R3) and let $Z \sim \mathcal{N}(0,1)$. 
Then for any function $h$ that is absolutely continuous and bounded,

$$
\begin{aligned}
& \left|\mathrm{E}\left[h\left(\sqrt{n i\left(\theta_{0}\right)}\left(\hat{\theta}_{n}(\boldsymbol{X})-\theta_{0}\right)\right)\right]-\mathrm{E}[h(Z)]\right| \\
& \leq \frac{\left\|h^{\prime}\right\|}{\sqrt{n}}\left(2+\frac{1}{\left[i\left(\theta_{0}\right)\right]^{\frac{3}{2}}} \mathrm{E}\left[\left|\left(\frac{\mathrm{d}}{\mathrm{d} \theta} \log f\left(X_{1} \mid \theta\right)\right)_{\theta=\theta_{0}}\right|^{3}\right]\right) \\
& +2\|h\| \frac{\mathrm{E}\left[\left(\hat{\theta}_{n}(\boldsymbol{X})-\theta_{0}\right)^{2}\right]}{\epsilon^{2}}+\frac{\left\|h^{\prime}\right\|}{\sqrt{n i\left(\theta_{0}\right)}}\left\{\mathrm{E}\left[\left|R_{2}\left(\theta_{0} ; \boldsymbol{X}\right)\right||| \hat{\theta}_{n}(\boldsymbol{X})-\theta_{0} \mid \leq \epsilon\right]\right. \\
& \left.+\frac{1}{2}\left[\mathrm{E}\left[\left(\sup _{\theta:\left|\theta-\theta_{0}\right| \leq \epsilon}\left|l^{(3)}(\theta ; \boldsymbol{X})\right|\right)^{2}|| \hat{\theta}_{n}(\boldsymbol{X})-\theta_{0} \mid \leq \epsilon\right]\right]^{\frac{1}{2}}\left[\mathrm{E}\left[\left(\hat{\theta}_{n}(\boldsymbol{X})-\theta_{0}\right)^{4}\right]\right]^{\frac{1}{2}}\right\},
\end{aligned}
$$

where

$$
R_{2}\left(\theta_{0} ; \boldsymbol{x}\right)=\left(\hat{\theta}_{n}(\boldsymbol{x})-\theta_{0}\right)\left(l^{\prime \prime}\left(\theta_{0} ; \boldsymbol{x}\right)+n i\left(\theta_{0}\right)\right) .
$$

Obvious observations are that the AR-bound requires finiteness of the fourth moment of $\hat{\theta}_{n}(\boldsymbol{X})-\theta_{0}$ and that this bound is more complicated than ours. Let us now comment on the bounds term by term.

- In the first term of the bounds, the different positioning of the expected Fisher information number is explained by the fact that we apply Lemma 2.1 to the standardised version of $g\left(X_{1}\right), g\left(X_{2}\right), \ldots, g\left(X_{n}\right)$, which have variance $\frac{\left[q^{\prime}\left(\theta_{0}\right)\right]^{2}}{i\left(\theta_{0}\right)}$, while Anastasiou and Reinert (2017) obtain the result by applying the lemma after standardising $\left.\frac{\mathrm{d}}{\mathrm{d} \theta} \log f\left(X_{1} \mid \theta\right)\right|_{\theta=\theta_{0}}$, $\left.\frac{\mathrm{d}}{\mathrm{d} \theta} \log f\left(X_{2} \mid \theta\right)\right|_{\theta=\theta_{0}}, \ldots,\left.\frac{\mathrm{d}}{\mathrm{d} \theta} \log f\left(X_{n} \mid \theta\right)\right|_{\theta=\theta_{0}}$, which have variance equal to $i\left(\theta_{0}\right)$.

- The second and third terms vanish in our bound when $q(\theta)=\theta \forall \theta \in \Theta$, while the AR-bound does not take this simplification into account. In addition, when $q(\theta) \neq \theta$ the second term is the same in both bounds, whereas the third term in our bound reads

$$
\mathrm{E}\left[\left(\hat{\theta}_{n}(\boldsymbol{X})-\theta_{0}\right)^{2}\right] \frac{\left\|h^{\prime}\right\| \sqrt{n i\left(\theta_{0}\right)}}{2\left|q^{\prime}\left(\theta_{0}\right)\right|} \sup _{\theta:\left|\theta-\theta_{0}\right| \leq \epsilon}\left|q^{\prime \prime}(\theta)\right|
$$

and is to be compared to

$$
\begin{aligned}
& \frac{\left\|h^{\prime}\right\|}{2 \sqrt{n i\left(\theta_{0}\right)}}\left[\mathrm{E}\left[\left(\sup _{\substack{\theta: \\
\left|\theta-\theta_{0}\right| \leq \epsilon}}\left|l^{(3)}(\theta ; \boldsymbol{X})\right|\right)^{2}|| \hat{\theta}_{n}(\boldsymbol{X})-\theta_{0} \mid \leq \epsilon\right]\right]^{\frac{1}{2}}\left[\mathrm{E}\left[\left(\hat{\theta}_{n}(\boldsymbol{X})-\theta_{0}\right)^{4}\right]\right]^{\frac{1}{2}} \\
& +\frac{\left\|h^{\prime}\right\|}{\sqrt{n i\left(\theta_{0}\right)}} \mathrm{E}\left[\left|R_{2}\left(\theta_{0} ; \boldsymbol{X}\right)\right|\left|\hat{\theta}_{n}(\boldsymbol{X})-\theta_{0}\right| \leq \epsilon\right]
\end{aligned}
$$

where $R_{2}\left(\theta_{0} ; \boldsymbol{x}\right)=\left(\hat{\theta}_{n}(\boldsymbol{x})-\theta_{0}\right)\left(l^{\prime \prime}\left(\theta_{0} ; \boldsymbol{x}\right)+n i\left(\theta_{0}\right)\right)$. The second derivative, $q^{\prime \prime}(\theta)$, plays in our bound the role of $l^{(3)}(\theta ; \boldsymbol{X})$, up to an important difference: $l^{(3)}(\theta ; \boldsymbol{X})$ is a sum. Consequently, the first term in $(2.15)$ has $\sqrt{n}$ in its numerator, exactly as in (2.14). The distinct positioning of the information quantity $i\left(\theta_{0}\right)$ has the same reason as explained above. Besides the 
obvious additional term in the AR bound (the second term in (2.15)), our bound is also clearly sharper at the level of moments of $\hat{\theta}_{n}(\boldsymbol{X})-\theta_{0}$ since

$$
\mathrm{E}\left[\left(\hat{\theta}_{n}(\boldsymbol{X})-\theta_{0}\right)^{2}\right] \leq\left[\mathrm{E}\left[\left(\hat{\theta}_{n}(\boldsymbol{X})-\theta_{0}\right)^{4}\right]\right]^{\frac{1}{2}}
$$

by the Cauchy-Schwarz inequality.

From this comparison one sees that our new bound is simpler and, moreover, has one term less. This is particularly striking in the simplest possible setting where $\hat{\theta}_{n}(\boldsymbol{X})$ is a sum of i.i.d. terms, where our bound clearly improves on the AR-bound. An advantage of the AR-bound is its wider applicability as it works for all MLE settings, even when an analytic expression of the MLE is not known.

We conclude this section by indicating practical statistical applications of our bounds in (2.1) and (2.2). This will be shown through their usefulness in the construction of confidence intervals for $\theta_{0}$. The Kolmogorov distance relates directly to exact conservative confidence intervals and the next proposition links our results to the Kolmogorov distance. Slightly different results for conservative confidence intervals were also given in Anastasiou and Reinert (2017).

Proposition 2.5. Let $W$ be any real-valued random variable and $Z \sim \mathcal{N}(0,1)$. Assume that there exist $\delta_{1}>0$ and $\delta_{2} \geq 0$ such that, for any function $h$ with $\left\|h^{\prime}\right\|$ and $\|h\|$ bounded,

$$
|\mathrm{E}[h(W)]-\mathrm{E}[h(Z)]| \leq \delta_{1}\left\|h^{\prime}\right\|+\delta_{2}\|h\| .
$$

Then $d_{K}(W, Z) \leq 2 \sqrt{\delta_{1}}+\delta_{2}$.

Proof: The proof of this proposition follows the approach explained in the proof of Theorem 3.3 on page 48 of Chen et al. (2011), where a similar result is shown that links the Wasserstein and the Kolmogorov distance. Let $z \in \mathbb{R}$ and for $\alpha=$ $\sqrt{\delta_{1}}(2 \pi)^{\frac{1}{4}}$ let

$$
h_{\alpha}(w)= \begin{cases}1, & \text { if } w \leq z, \\ 1+\frac{z-w}{\alpha}, & \text { if } z<w \leq z+\alpha, \\ 0, & \text { if } w>z+\alpha,\end{cases}
$$

so that $h_{\alpha}$ is bounded Lipschitz with $\left\|h_{\alpha}\right\| \leq 1$ and $\left\|h_{\alpha}^{\prime}\right\| \leq \frac{1}{\alpha}$. The definition of $h_{\alpha}$ combined with (2.16) leads to

$$
\begin{aligned}
\mathbb{P}(W \leq z)-\mathbb{P}(Z \leq z) & \leq \mathrm{E}\left[h_{\alpha}(W)\right]-\mathrm{E}\left[h_{\alpha}(Z)\right]+\mathrm{E}\left[h_{\alpha}(Z)\right]-\mathbb{P}(Z \leq z) \\
& \leq\left|\mathrm{E}\left[h_{\alpha}(W)\right]-\mathrm{E}\left[h_{\alpha}(Z)\right]\right|+\mathrm{E}\left[h_{\alpha}(Z)\right]-\mathbb{P}(Z \leq z) \\
& \leq \delta_{1}\left\|h_{\alpha}^{\prime}\right\|+\delta_{2}\left\|h_{\alpha}\right\|+\mathbb{P}(z<Z \leq z+\alpha) \\
& \leq \frac{\delta_{1}}{\alpha}+\delta_{2}+\frac{\alpha}{\sqrt{2 \pi}} \\
& =2 \frac{\sqrt{\delta_{1}}}{(2 \pi)^{\frac{1}{4}}}+\delta_{2} \leq 2 \sqrt{\delta_{1}}+\delta_{2} .
\end{aligned}
$$

Similarly, using now

$$
h_{\alpha}^{*}(w)= \begin{cases}0, & \text { if } w \leq z-\alpha, \\ \frac{w-(z-\alpha)}{\alpha}, & \text { if } z-\alpha<w \leq z, \\ 1, & \text { if } w>z\end{cases}
$$


we can show that $\mathbb{P}(W \leq z)-\mathbb{P}(Z \leq z) \geq-\left(2 \sqrt{\delta_{1}}+\delta_{2}\right)$, which completes the proof.

Our bounds are of the form (2.16), where in our case $W=\sqrt{n i\left(\theta_{0}\right)}\left(\hat{\theta}_{n}(\boldsymbol{X})-\theta_{0}\right)$ and $\delta_{1}$ and $\delta_{2}$ are explicitly given with $\delta_{1}=\mathcal{O}\left(\frac{1}{\sqrt{n}}\right)$ and $\delta_{2}=\mathcal{O}\left(\frac{1}{n}\right)$ in (2.1), while for (2.2) take $\delta_{2}=0$. Using Proposition 2.5 we get

$$
d_{K}\left(\sqrt{n i\left(\theta_{0}\right)}\left(\hat{\theta}_{n}(\boldsymbol{X})-\theta_{0}\right), Z\right) \leq 2 \sqrt{\delta_{1}}+\delta_{2} \leq B_{K},
$$

where $B_{K}$ is a sharp bound not depending on $\theta_{0}$ or the data. In view of Theorem 2.2, this bound can be obtained by upper bounding $1 / q^{\prime}\left(\theta_{0}\right)$ and $\sup _{\theta:\left|\theta-\theta_{0}\right| \leq \epsilon}\left|q^{\prime \prime}(\theta)\right|$ (which is done on a case-by-case basis) as well as the mean squared error $\mathrm{E}\left[\left(\hat{\theta}_{n}(\boldsymbol{X})-\theta_{0}\right)^{2}\right]$ (e.g., through Theorem 5.1 in Anastasiou and Reinert, 2017). The presence of the Fisher information quantity is in many settings not a problem, as it often does not depend on $\theta_{0}$; this is typically the case for location models. Therefore, for $y \in \mathbb{R}$ we have

$$
\begin{aligned}
& \left|\mathbb{P}\left(\sqrt{n i\left(\theta_{0}\right)}\left(\hat{\theta}_{n}(\boldsymbol{X})-\theta_{0}\right) \leq y\right)-\mathbb{P}(Z \leq y)\right| \leq B_{K} \\
& \Leftrightarrow-B_{K} \leq \mathbb{P}\left(\sqrt{n i\left(\theta_{0}\right)}\left(\hat{\theta}_{n}(\boldsymbol{X})-\theta_{0}\right) \leq y\right)-\mathbb{P}(Z \leq y) \leq B_{K} .
\end{aligned}
$$

With $\Phi^{-1}(\cdot)$ the quantile function of the standard normal distribution, $\alpha \in[0,1]$ and $\frac{\alpha}{2}-B_{K}>0$, applying (2.17) to $y=\Phi^{-1}\left(\frac{\alpha}{2}-B_{K}\right)$ and to $y=$ $\Phi^{-1}\left(1-\frac{\alpha}{2}+B_{K}\right)$ yields

$$
\mathbb{P}\left(\Phi^{-1}\left(\frac{\alpha}{2}-B_{K}\right) \leq \sqrt{n i\left(\theta_{0}\right)}\left(\hat{\theta}_{n}(\boldsymbol{X})-\theta_{0}\right) \leq \Phi^{-1}\left(1-\frac{\alpha}{2}+B_{K}\right)\right) \geq 1-\alpha .
$$

Hence, if the expected Fisher information number for one random variable, $i\left(\theta_{0}\right)$, is known and does not depend on $\theta_{0}$, then

$$
\left(\hat{\theta}_{n}(\boldsymbol{X})-\frac{\Phi^{-1}\left(1-\frac{\alpha}{2}+B_{K}\right)}{\sqrt{n i\left(\theta_{0}\right)}}, \hat{\theta}_{n}(\boldsymbol{X})-\frac{\Phi^{-1}\left(\frac{\alpha}{2}-B_{K}\right)}{\sqrt{n i\left(\theta_{0}\right)}}\right)
$$

is a conservative $100(1-\alpha) \%$ confidence interval for $\theta_{0}$.

\section{Calculation of the bound in different scenarios}

In this section we shall consider examples for which we explicitly calculate our upper bounds from Theorem 2.2 and compare them to the AR-bound. In particular, we shall show that our results are tailor-made for one-parameter exponential families. To further assess their accuracy, we simulate data from exponential distributions and compare our corresponding bound to the actual distance between the unknown exact law of the MLE and its asymptotic normal law, for distinct values of the sample size $n$.

3.1. Bounds for one-parameter exponential families. The probability density (or mass) function for one-parameter exponential families is given by

$$
f(x \mid \theta)=\exp \{k(\theta) T(x)-A(\theta)+S(x)\} \mathbb{1}_{\{x \in B\}},
$$

where the set $B=\{x: f(x \mid \theta)>0\}$ is the support of the density and does not depend on $\theta ; k(\theta)$ and $A(\theta)$ are functions of the parameter; $T(x)$ and $S(x)$ are 
functions only of the data. Whenever $k(\theta)=\theta$ we have the so-called canonical case, where $\theta$ and $T(X)$ are called the natural parameter and natural observation (Casella and Berger, 1990). The identifiability constraint in (R1) entails that $k^{\prime}(\theta) \neq 0$ (Geyer, 2013), an important detail for the following investigation.

Proposition 3.1. Suppose $X_{1}, \ldots, X_{n}$ are i.i.d. with probability density (or mass) function that can be expressed in the form of (3.1). Assume that the regularity conditions (R1)-(R4) are satisfied and the $M L E \hat{\theta}_{n}(\boldsymbol{X})$ exists and is unique. Let $\theta \mapsto D(\theta)=\frac{A^{\prime}(\theta)}{k^{\prime}(\theta)}$ be invertible. Then $q(\cdot)=D(\cdot)$, with $q: \Theta \rightarrow \mathbb{R}$ as in Theorem 2.2. Moreover, under the same regularity conditions, no distributions other than (3.1) admit an MLE of the form (1.3).

Proof: Using (3.1), we have that

$$
\begin{aligned}
& L(\theta ; \boldsymbol{x})=\prod_{i=1}^{n} f\left(x_{i} \mid \theta\right)=\exp \left\{k(\theta) \sum_{i=1}^{n} T\left(x_{i}\right)-n A(\theta)+\sum_{i=1}^{n} S\left(x_{i}\right)\right\}, \\
& l(\theta ; \boldsymbol{x})=k(\theta) \sum_{i=1}^{n} T\left(x_{i}\right)-n A(\theta)+\sum_{i=1}^{n} S\left(x_{i}\right),
\end{aligned}
$$

and hence

$$
l^{\prime}(\theta ; \boldsymbol{x})=k^{\prime}(\theta) \sum_{i=1}^{n} T\left(x_{i}\right)-n A^{\prime}(\theta)=0 \Longleftrightarrow D(\theta)=\frac{1}{n} \sum_{i=1}^{n} T\left(x_{i}\right),
$$

which means that $\hat{\theta}_{n}(\boldsymbol{X})=D^{-1}\left(\frac{1}{n} \sum_{i=1}^{n} T\left(X_{i}\right)\right)$ under the invertibility assumption for $D(\theta)$. It follows that $q(\cdot)=D(\cdot)$.

For the second statement, let us start from $q\left(\hat{\theta}_{n}(\boldsymbol{X})\right)=\frac{1}{n} \sum_{i=1}^{n} g\left(X_{i}\right)$. Such an MLE form necessarily comes from a log-likelihood equation of the form $q(\theta)=$ $\frac{1}{n} \sum_{i=1}^{n} g\left(x_{i}\right) \Longleftrightarrow \sum_{i=1}^{n} g\left(x_{i}\right)-n q(\theta)=0$. Let us write the invertible function $q(\theta)$ as some ratio $\alpha^{\prime}(\theta) / \beta^{\prime}(\theta)$ for two differentiable functions $\alpha$ and $\beta$ with $\beta^{\prime}(\theta) \neq 0 \forall \theta \in \Theta$. From this we deduce that $l(\theta ; \boldsymbol{x})=\beta(\theta) \sum_{i=1}^{n} g\left(x_{i}\right)-n \alpha(\theta)+f(\boldsymbol{x})$ for some function $f$ of the observations. Since $l(\theta ; \boldsymbol{x})$ is a log-likelihood built from independent observations, the function $f$ necessarily is a sum which we write $\sum_{i=1}^{n} S\left(x_{i}\right)$ (which could also contain constants). The claim now readily follows.

This result hence shows that, as announced in the Introduction, the broad oneparameter exponential families do satisfy (1.3) and there is no distribution outside these families that can be of that form under the given regularity conditions. This remarkable result represents a new characterization of the exponential families through their MLE. With this in hand, Theorem 2.2 can be applied to (3.1), resulting in

Corollary 3.2. Let $X_{1}, \ldots, X_{n}$ be i.i.d. random variables with the probability density (or mass) function of a one-parameter exponential family. Assume that (R1)-(R4) are satisfied and the $M L E \hat{\theta}_{n}(\boldsymbol{X})$ exists and is unique. Then, with $Z \sim \mathcal{N}(0,1)$ and $h \in H$ as defined in $(1.2)$, 
(1) if $D^{\prime \prime}(\cdot)$ is not uniformly bounded but for any $\theta_{0} \in \Theta$ there exists $0<\epsilon=$ $\epsilon\left(\theta_{0}\right)$ such that $\sup _{\theta:\left|\theta-\theta_{0}\right|<\epsilon}\left|D^{\prime \prime}(\theta)\right|<\infty$, we have

$$
\begin{aligned}
& \left|\mathrm{E}\left[h\left(\sqrt{n i\left(\theta_{0}\right)}\left(\hat{\theta}_{n}(\boldsymbol{X})-\theta_{0}\right)\right)\right]-\mathrm{E}[h(Z)]\right| \\
& \leq \frac{\left\|h^{\prime}\right\|}{\sqrt{n}}\left(2+\frac{\left|k^{\prime}\left(\theta_{0}\right)\right|^{3} \mathrm{E}\left[\left|T\left(X_{1}\right)-D\left(\theta_{0}\right)\right|^{3}\right]}{\left|A^{\prime \prime}\left(\theta_{0}\right)-k^{\prime \prime}\left(\theta_{0}\right) D\left(\theta_{0}\right)\right|^{\frac{3}{2}}}\right) \\
& +\mathrm{E}\left[\left(\hat{\theta}_{n}(\boldsymbol{X})-\theta_{0}\right)^{2}\right]\left(2 \frac{\|h\|}{\epsilon^{2}} \mathbb{1}\{\exists \theta \in \Theta: D(\theta) \neq \theta\}\right. \\
& \left.\quad+\frac{\left\|h^{\prime}\right\| \sqrt{n}\left|k^{\prime}\left(\theta_{0}\right)\right|}{2 \sqrt{\left|A^{\prime \prime}\left(\theta_{0}\right)-k^{\prime \prime}\left(\theta_{0}\right) D\left(\theta_{0}\right)\right|}} \sup _{\theta:\left|\theta-\theta_{0}\right| \leq \epsilon}\left|D^{\prime \prime}(\theta)\right|\right) ;
\end{aligned}
$$

(2) if $D^{\prime \prime}(\cdot)$ is uniformly bounded, with $\left|D^{\prime \prime}(\theta)\right| \leq B^{*} \forall \theta \in \Theta$ for some $B^{*}>0$, we have

$$
\begin{aligned}
& \left|\mathrm{E}\left[h\left(\sqrt{n i\left(\theta_{0}\right)}\left(\hat{\theta}_{n}(\boldsymbol{X})-\theta_{0}\right)\right)\right]-\mathrm{E}[h(Z)]\right| \\
& \leq \frac{\left\|h^{\prime}\right\|}{\sqrt{n}}\left(2+\frac{\left|k^{\prime}\left(\theta_{0}\right)\right|^{3} \mathrm{E}\left[\left|T\left(X_{1}\right)-D\left(\theta_{0}\right)\right|^{3}\right]}{\left|A^{\prime \prime}\left(\theta_{0}\right)-k^{\prime \prime}\left(\theta_{0}\right) D\left(\theta_{0}\right)\right|^{\frac{3}{2}}}\right) \\
& +\left\|h^{\prime}\right\| B^{*} \frac{\sqrt{n}\left|k^{\prime}\left(\theta_{0}\right)\right|}{2 \sqrt{\left|A^{\prime \prime}\left(\theta_{0}\right)-k^{\prime \prime}\left(\theta_{0}\right) D\left(\theta_{0}\right)\right|}} \mathrm{E}\left[\left(\hat{\theta}_{n}(\boldsymbol{X})-\theta_{0}\right)^{2}\right] \mathbb{1}\{\exists \theta \in \Theta: D(\theta) \neq \theta\} .
\end{aligned}
$$

Proof: We readily have

$i\left(\theta_{0}\right)=\mathrm{E}\left[-l^{\prime \prime}\left(\theta_{0} ; X_{1}\right)\right]=A^{\prime \prime}\left(\theta_{0}\right)-k^{\prime \prime}\left(\theta_{0}\right) \mathrm{E}\left[T\left(X_{1}\right)\right]=\frac{A^{\prime \prime}\left(\theta_{0}\right) k^{\prime}\left(\theta_{0}\right)-k^{\prime \prime}\left(\theta_{0}\right) A^{\prime}\left(\theta_{0}\right)}{k^{\prime}\left(\theta_{0}\right)}$

and $q^{\prime}\left(\theta_{0}\right)=\frac{A^{\prime \prime}\left(\theta_{0}\right) k^{\prime}\left(\theta_{0}\right)-k^{\prime \prime}\left(\theta_{0}\right) A^{\prime}\left(\theta_{0}\right)}{\left[k^{\prime}\left(\theta_{0}\right)\right]^{2}}$. Combining these two results yields

$$
\frac{\sqrt{i\left(\theta_{0}\right)}}{\left|q^{\prime}\left(\theta_{0}\right)\right|}=\frac{\left|k^{\prime}\left(\theta_{0}\right)\right|^{\frac{3}{2}}}{\sqrt{\left|A^{\prime \prime}\left(\theta_{0}\right) k^{\prime}\left(\theta_{0}\right)-k^{\prime \prime}\left(\theta_{0}\right) A^{\prime}\left(\theta_{0}\right)\right|}}=\frac{\left|k^{\prime}\left(\theta_{0}\right)\right|}{\sqrt{\left|A^{\prime \prime}\left(\theta_{0}\right)-k^{\prime \prime}\left(\theta_{0}\right) D\left(\theta_{0}\right)\right|}} .
$$

This result, along with the fact that $g(x)=T(x)$ and $q(\theta)=D(\theta)$ by Proposition 3.1, allows to deduce the announced upper bound from Theorem 2.2.

Remark 3.3. It is particularly interesting to spell out this bound in the canonical case $k(\theta)=\theta$. Since then $k^{\prime}(\theta)=1, D(\theta)=A^{\prime}(\theta)$, we find that if $A^{\prime \prime \prime}(\cdot)$ is not uniformly bounded but for any $\theta_{0} \in \Theta$ there exists $0<\epsilon=\epsilon\left(\theta_{0}\right)$ such that 


$$
\begin{aligned}
& \sup _{\theta:\left|\theta-\theta_{0}\right|<\epsilon}\left|A^{\prime \prime \prime}(\theta)\right|<\infty, \text { then } \\
& \qquad\left|\mathrm{E}\left[h\left(\sqrt{n i\left(\theta_{0}\right)}\left(\hat{\theta}_{n}(\boldsymbol{X})-\theta_{0}\right)\right)\right]-\mathrm{E}[h(Z)]\right| \\
& \leq \frac{\left\|h^{\prime}\right\|}{\sqrt{n}}\left(2+\frac{\mathrm{E}\left[\left|T\left(X_{1}\right)-A^{\prime}\left(\theta_{0}\right)\right|^{3}\right]}{\left|A^{\prime \prime}\left(\theta_{0}\right)\right|^{\frac{3}{2}}}\right) \\
& +\mathrm{E}\left[\left(\hat{\theta}_{n}(\boldsymbol{X})-\theta_{0}\right)^{2}\right]\left(2 \frac{\|h\|}{\epsilon^{2}} \mathbb{1}\left\{\exists \theta \in \Theta: A^{\prime}(\theta) \neq \theta\right\}\right. \\
& \left.\quad+\frac{\left\|h^{\prime}\right\| \sqrt{n}}{2 \sqrt{\left|A^{\prime \prime}\left(\theta_{0}\right)\right|}} \sup _{\theta:\left|\theta-\theta_{0}\right| \leq \epsilon}\left|A^{\prime \prime \prime}(\theta)\right|\right) .
\end{aligned}
$$

A similar result as the one in (3.3) holds when $A^{\prime \prime \prime}(\cdot)$ is uniformly bounded. Now, as $i(\theta)=A^{\prime \prime}(\theta)$ and $l^{\prime \prime}(\theta ; \boldsymbol{X})=-n A^{\prime \prime}(\theta), R_{2}(\theta ; \boldsymbol{x})=0$ and straightforward manipulations show that all terms in the AR-bound coincide with those in our bound (3.2), except for $\left[\mathrm{E}\left[\left(\hat{\theta}_{n}(\boldsymbol{X})-\theta_{0}\right)^{4}\right]\right]^{\frac{1}{2}}$, making the AR-bound less sharp than ours. However, Anastasiou and Reinert (2017) have shown that, in the canonical exponential setting, their bound can actually have an $\mathrm{E}\left[\left(\hat{\theta}_{n}(\boldsymbol{X})-\theta_{0}\right)^{2}\right]$ factor, implying that both bounds are exactly the same in the canonical case. In order to get an idea of how our bound improves on the AR-bound in non-canonical cases, we treat the exponential distribution under a non-canonical parametrisation in subsection 3.3 .

3.2. Bounds for the Generalised Gamma distribution. Let us consider $X_{1}, \ldots, X_{n}$ i.i.d. random variables from the Generalized Gamma $\operatorname{GG}(\theta, d, p)$ distribution, where the shape parameters $d, p>0$ are considered to be known and the scale parameter $\theta$ is the unknown parameter of interest. The Generalised Gamma distribution includes many other known distributions as special cases: the Weibull for $d=p$, the Gamma when $p=1$, and the negative exponential when $p=d=1$. Indeed, with $\Gamma(\cdot)$ denoting the Gamma function, the probability density function for $x>0$ is

$$
\begin{aligned}
f(x \mid \theta) & =\frac{p x^{d-1} \exp \left\{-\left(\frac{x}{\theta}\right)^{p}\right\}}{\theta^{d}} \frac{\Gamma\left(\frac{d}{p}\right)}{} \\
& =\exp \left\{-\frac{x^{p}}{\theta^{p}}+\log p-d \log \theta+(d-1) \log x-\log \left(\Gamma\left(\frac{d}{p}\right)\right)\right\}
\end{aligned}
$$

where, in the terminology of one-parameter exponential families, $B=(0, \infty), \Theta=$ $(0, \infty), T(x)=x^{p}, k(\theta)=-\frac{1}{\theta^{p}}, A(\theta)=d \log \theta$ and $S(x)=\log p+(d-1) \log x-$ $\log \left(\Gamma\left(\frac{d}{p}\right)\right)$. Simple steps yield

$$
\begin{aligned}
& l\left(\theta_{0} ; \boldsymbol{x}\right)=-\frac{1}{\theta_{0}^{p}} \sum_{i=1}^{n} x_{i}^{p}+n \log p-n d \log \theta_{0}+(d-1) \log \left(\prod_{i=1}^{n} x_{i}\right)-n \log \left(\Gamma\left(\frac{d}{p}\right)\right) \\
& l^{\prime}\left(\theta_{0} ; \boldsymbol{x}\right)=\frac{p}{\theta_{0}^{p+1}} \sum_{i=1}^{n} x_{i}^{p}-n \frac{d}{\theta_{0}}=0 \Leftrightarrow \hat{\theta}_{n}(\boldsymbol{x})=\left(\frac{p}{n d} \sum_{i=1}^{n} x_{i}^{p}\right)^{\frac{1}{p}} .
\end{aligned}
$$


It is easy to verify that indeed $l^{\prime \prime}\left(\hat{\theta}_{n}(\boldsymbol{x}) ; \boldsymbol{x}\right)=-n \frac{p d}{\left[\hat{\theta}_{n}(\boldsymbol{x})\right]^{2}}<0$, which shows that the MLE exists and is unique. The regularity conditions (R1)-(R4) are also satisfied and $D\left(\theta_{0}\right)=q\left(\theta_{0}\right)=\frac{d}{p} \theta_{0}^{p}$ with its second derivative not being uniformly bounded but bounded in a neighbourhood of $\theta_{0}$. Using therefore the first result of Corollary 3.2 for $\epsilon=\frac{\theta_{0}}{2}$ we obtain

$$
\begin{aligned}
& \left|\mathrm{E}\left[h\left(\sqrt{n i\left(\theta_{0}\right)}\left(\hat{\theta}_{n}(\boldsymbol{X})-\theta_{0}\right)\right)\right]-\mathrm{E}[h(Z)]\right| \leq \frac{\left\|h^{\prime}\right\|}{\sqrt{n}}\left(2+\left(3+6 \frac{p}{d}\right)^{\frac{3}{4}}\right) \\
& +\left(1-2\left(\frac{p}{n d}\right)^{\frac{1}{p}} \frac{\Gamma\left(\frac{n d+1}{p}\right)}{\Gamma\left(\frac{n d}{p}\right)}+\left(\frac{p}{n d}\right)^{\frac{2}{p}} \frac{\Gamma\left(\frac{n d+2}{p}\right)}{\Gamma\left(\frac{n d}{p}\right)}\right) \mathbb{1}\{\{d \neq 1\} \cup\{p \neq 1\}\} \\
& \times\left[8\|h\|+\frac{\left\|h^{\prime}\right\| \sqrt{n d p}|p-1|}{2}\left(\frac{1}{2^{p-2}} \mathbb{1}\{p<2\}+\left(\frac{3}{2}\right)^{p-2} \mathbb{1}\{p \geq 2\}\right)\right] .
\end{aligned}
$$

Let us briefly show how to obtain this bound. As already indicated, for the Generalised Gamma distribution, $D\left(\theta_{0}\right)=\frac{d}{p} \theta_{0}^{p}$ and thus $\mathrm{E}\left[\left|T(X)-D\left(\theta_{0}\right)\right|^{3}\right]=$ $\mathrm{E}\left[\left|X^{p}-\frac{d}{p} \theta_{0}^{p}\right|^{3}\right]$. This third absolute moment is very complicated to calculate. Therefore, we use Hölder's inequality and the fact that $X \sim \operatorname{GG}\left(\theta_{0}, d, p\right)$ and thus $X^{p} \sim \operatorname{Gamma}\left(\frac{d}{p}, \frac{1}{\theta_{0}^{p}}\right)$ to get

$$
\begin{aligned}
& \mathrm{E}\left[\left|X^{p}-\frac{d}{p} \theta_{0}^{p}\right|^{3}\right] \leq\left[\mathrm{E}\left[\left(X^{p}-\frac{d}{p} \theta_{0}^{p}\right)^{4}\right]\right]^{\frac{3}{4}} \\
& =\left[\mathrm{E}\left[X^{4 p}\right]+\left(\frac{d \theta_{0}^{p}}{p}\right)^{4}+6\left(\frac{d \theta_{0}^{p}}{p}\right)^{2} \mathrm{E}\left[X^{2 p}\right]-4\left(\frac{d \theta_{0}^{p}}{p}\right)^{3} \mathrm{E}\left[X^{p}\right]-4 \frac{d}{p} \theta_{0}^{p} \mathrm{E}\left[X^{3 p}\right]\right]^{\frac{3}{4}} \\
& =\left[\theta_{0}^{4 p} \frac{d}{p}\left(6+3 \frac{d}{p}\right)\right]^{\frac{3}{4}}=\theta_{0}^{3 p}\left(\frac{d}{p}\right)^{\frac{3}{4}}\left(6+3 \frac{d}{p}\right)^{\frac{3}{4}}
\end{aligned}
$$

Simpler calculations yield

$$
\frac{\left|k^{\prime}\left(\theta_{0}\right)\right|}{\sqrt{\left|A^{\prime \prime}\left(\theta_{0}\right)-k^{\prime \prime}\left(\theta_{0}\right) D\left(\theta_{0}\right)\right|}}=\frac{\sqrt{p}}{\sqrt{d} \theta_{0}^{p}} .
$$

Using that $X_{i}^{p} \sim \operatorname{Gamma}\left(\frac{d}{p}, \frac{1}{\theta_{0}^{p}}\right) \Rightarrow \sum_{i=1}^{n} X_{i}^{p} \sim \operatorname{Gamma}\left(n \frac{d}{p}, \frac{1}{\theta_{0}^{p}}\right)$, we get

$$
\begin{aligned}
& \mathrm{E}\left[\left(\hat{\theta}_{n}(\boldsymbol{X})-\theta_{0}\right)^{2}\right] \\
& =\left(\frac{p}{n d}\right)^{\frac{2}{p}} \mathrm{E}\left[\left(\sum_{i=1}^{n} X_{i}^{p}\right)^{\frac{2}{p}}\right]+\theta_{0}^{2}-2\left(\frac{p}{n d}\right)^{\frac{1}{p}} \theta_{0} \mathrm{E}\left[\left(\sum_{i=1}^{n} X_{i}^{p}\right)^{\frac{1}{p}}\right] \\
& =\theta_{0}^{2}\left(\frac{p}{n d}\right)^{\frac{2}{p}} \frac{\Gamma\left(\frac{n d+2}{p}\right)}{\Gamma\left(\frac{n d}{p}\right)}+\theta_{0}^{2}-2 \theta_{0}^{2}\left(\frac{p}{n d}\right)^{\frac{1}{p}} \frac{\Gamma\left(\frac{n d+1}{p}\right)}{\Gamma\left(\frac{n d}{p}\right)}
\end{aligned}
$$




$$
=\theta_{0}^{2}\left(1-2\left(\frac{p}{n d}\right)^{\frac{1}{p}} \frac{\Gamma\left(\frac{n d+1}{p}\right)}{\Gamma\left(\frac{n d}{p}\right)}+\left(\frac{p}{n d}\right)^{\frac{2}{p}} \frac{\Gamma\left(\frac{n d+2}{p}\right)}{\Gamma\left(\frac{n d}{p}\right)}\right) .
$$

The second derivative of the function $D(\theta)$ is not uniformly bounded and thus we use the result in (3.2). Regarding $\sup _{\theta:\left|\theta-\theta_{0}\right| \leq \epsilon}\left|D^{\prime \prime}(\theta)\right|$, one has to be careful as the supremum depends on the value of $p$ :

$$
\begin{aligned}
\sup _{\theta:\left|\theta-\theta_{0}\right| \leq \epsilon}\left|d(p-1) \theta^{p-2}\right| & =d|p-1| \sup _{\theta:\left|\theta-\theta_{0}\right| \leq \epsilon}\left|\theta^{p-2}\right| \\
& =d|p-1| \begin{cases}\left(\theta_{0}-\epsilon\right)^{p-2}, & \text { if } 0<p<2 \\
\left(\theta_{0}+\epsilon\right)^{p-2}, & \text { if } p \geq 2 .\end{cases}
\end{aligned}
$$

Thus, applying now the results of (3.5), (3.6), (3.7) and (3.8) on the general expression for the first upper bound in Corollary 3.2, we obtain the result in (3.4).

Remark 3.4. (1) The bound in (3.4) is $\mathcal{O}\left(\frac{1}{\sqrt{n}}\right)$. This is not obvious as we need to comment on the order of the term $\left(1-2\left(\frac{p}{n d}\right)^{\frac{1}{p}} \frac{\Gamma\left(\frac{n d+1}{p}\right)}{\Gamma\left(\frac{n d}{p}\right)}+\left(\frac{p}{n d}\right)^{\frac{2}{p}} \frac{\Gamma\left(\frac{n d+2}{p}\right)}{\Gamma\left(\frac{n d}{p}\right)}\right)$. Using the Taylor expansion for a ratio of Gamma functions (see Tricomi and Erdélyi, 1951)

$$
\frac{\Gamma(z+a)}{\Gamma(z+b)}=z^{a-b}\left(1+\frac{(a-b)(a+b-1)}{2 z}+O\left(|z|^{-2}\right)\right)
$$

for large $z$ (here, $n d / p)$ and bounded $a$ and $b$, we can see that this term is of order $\frac{1}{n}$, leading to the overall order of $\frac{1}{\sqrt{n}}$.

(2) The indicator function in the last term of the bound in (3.4) comes from the fact that $q(\theta)=\theta \forall \theta \in \Theta \Longleftrightarrow d, p=1$.

3.3. Bounds for the (negative) exponential distribution. In this subsection, we consider the most famous special case of the Generalised Gamma distribution: the (negative) exponential distribution. First we will treat the canonical form of the distribution and then we will change the parametrisation to discuss the more interesting non-canonical setting. In the canonical case $D^{\prime \prime}(\cdot)$ is not uniformly bounded but it is bounded in a neighbourhood of $\theta_{0}$; therefore, (3.2) of Corollary 3.2 is used. As for the non-canonical setting, we will show that $D(\theta)=\theta \forall \theta \in \Theta$ which means that only the (same) first term of the bounds in (3.2) and (3.3) survives.

3.3.1. The canonical case: $\operatorname{Exp}(\theta)$. We start with $X_{1}, \ldots, X_{n}$ exponentially distributed i.i.d. random variables with scale parameter $\theta>0$ and probability density function $f(x \mid \theta)=\theta \exp \{-\theta x\}=\exp \{\log \theta-\theta x\}$ for $x>0$, which we write $\operatorname{Exp}(\theta)$. In terms of (3.1), this means $B=(0, \infty), \Theta=(0, \infty), T(x)=-x, k(\theta)=\theta$, $A(\theta)=-\log \theta$ and $S(x)=0$. Further we have that

$$
l^{\prime}(\theta ; \boldsymbol{x})=\frac{n}{\theta}-\sum_{i=1}^{n} x_{i}, \quad l^{\prime \prime}(\theta ; \boldsymbol{x})=-\frac{n}{\theta^{2}},
$$

the unique MLE is given by $\hat{\theta}_{n}(\boldsymbol{X})=1 / \bar{X}$ with $\bar{X}=\frac{1}{n} \sum_{i=1}^{n} X_{i}$ and (R1)-(R4) are satisfied. 
With this in hand, we can easily see that $D\left(\theta_{0}\right)=q\left(\theta_{0}\right)=\frac{A^{\prime}\left(\theta_{0}\right)}{k^{\prime}\left(\theta_{0}\right)}=-\frac{1}{\theta_{0}}$ and

$$
\frac{\left|k^{\prime}\left(\theta_{0}\right)\right|}{\sqrt{\left|A^{\prime \prime}\left(\theta_{0}\right)-k^{\prime \prime}\left(\theta_{0}\right) D\left(\theta_{0}\right)\right|}}=\theta_{0} .
$$

Simple calculations allow us here to bypass the Hölder inequality used for the Generalized Gamma case and to obtain $\mathrm{E}\left[\left|T(X)-D\left(\theta_{0}\right)\right|^{3}\right]=\mathrm{E}\left[\left|\frac{1}{\theta_{0}}-X\right|^{3}\right] \leq \frac{2.41456}{\theta_{0}^{3}}$. Since $X_{i} \sim \operatorname{Exp}(\theta) \forall i \in\{1,2, \ldots, n\}$ then $\bar{X} \sim \operatorname{Gam}(n, n \theta)$, with $\operatorname{Gam}(\alpha, \beta)$ being the Gamma distribution with shape parameter $\alpha$ and rate parameter $\beta$. Consequently,

$$
\mathrm{E}\left[\left(\hat{\theta}_{n}(\boldsymbol{X})-\theta_{0}\right)^{2}\right]=\frac{\left(n \theta_{0}\right)^{2}}{(n-1)(n-2)}-\frac{2 n \theta_{0}^{2}}{n-1}+\theta_{0}^{2}=\frac{(n+2) \theta_{0}^{2}}{(n-1)(n-2)} .
$$

Moreover, for $\epsilon>0$ such that $0<\epsilon<\theta_{0}$, we obtain $\sup _{\theta:\left|\theta-\theta_{0}\right| \leq \epsilon}\left|D^{\prime \prime}(\theta)\right|=\frac{2}{\left(\theta_{0}-\epsilon\right)^{3}}$. Choosing $\epsilon=\frac{\theta_{0}}{2}$, we get $\sup _{\theta:\left|\theta-\theta_{0}\right| \leq \epsilon}\left|D^{\prime \prime}(\theta)\right|=\frac{16}{\theta_{0}^{3}}$. Using this result and (3.9), Corollary 3.2(1) gives

$$
\begin{aligned}
\left|\mathrm{E}\left[h\left(\sqrt{n i\left(\theta_{0}\right)}\left(\hat{\theta}_{n}(\boldsymbol{X})-\theta_{0}\right)\right)\right]-\mathrm{E}[h(Z)]\right| \leq & 4.41456 \frac{\left\|h^{\prime}\right\|}{\sqrt{n}}+8\|h\| \frac{(n+2)}{(n-1)(n-2)} \\
& +8\left\|h^{\prime}\right\| \frac{\sqrt{n}(n+2)}{(n-1)(n-2)} .
\end{aligned}
$$

This bound is of order $\mathcal{O}\left(\frac{1}{\sqrt{n}}\right)$ and coincides, as discussed in Remark 3.3, with the AR-bound.

3.3.2. The non-canonical case: $\operatorname{Exp}\left(\frac{1}{\theta}\right)$. We now proceed to examine the more interesting case where $X_{1}, \ldots, X_{n}$ are i.i.d. random variables from $\operatorname{Exp}\left(\frac{1}{\theta}\right)$. The probability density function is

$$
f(x \mid \theta)=\frac{1}{\theta} \exp \left\{-\frac{1}{\theta} x\right\}=\exp \left\{-\log \theta-\frac{1}{\theta} x\right\}
$$

corresponding to $B=(0, \infty), \Theta=(0, \infty), T(x)=x, k(\theta)=-\frac{1}{\theta}, A(\theta)=\log \theta$ and $S(x)=0$. As before, simple steps give that the MLE exists, it is unique and equal to $\hat{\theta}_{n}(\boldsymbol{X})=\bar{X}$. The regularity conditions are satisfied and we obtain using (3.2) ((3.3) will give the same result) that

$$
\left|\mathrm{E}\left[h\left(\sqrt{n i\left(\theta_{0}\right)}\left(\hat{\theta}_{n}(\boldsymbol{X})-\theta_{0}\right)\right)\right]-\mathrm{E}[h(Z)]\right| \leq 4.41456 \frac{\left\|h^{\prime}\right\|}{\sqrt{n}} .
$$

Indeed, $D\left(\theta_{0}\right)=q\left(\theta_{0}\right)=\theta_{0}$, making the last two terms of both bounds in Corollary 3.2 vanish. The result then follows from $\mathrm{E}\left[\left|T(X)-D\left(\theta_{0}\right)\right|^{3}\right]=\mathrm{E}\left[\left|X-\theta_{0}\right|^{3}\right] \leq$ $2.41456 \theta_{0}^{3}$ and

$$
\frac{\left|k^{\prime}\left(\theta_{0}\right)\right|}{\sqrt{\left|A^{\prime \prime}\left(\theta_{0}\right)-k^{\prime \prime}\left(\theta_{0}\right) D\left(\theta_{0}\right)\right|}}=\frac{1}{\theta_{0}} .
$$

Remark 3.5. (1) The order of the bound in terms of the sample size is, as expected, $\frac{1}{\sqrt{n}}$, corresponding to the order obtained for the Generalized Gamma distribution. The constant here is better than the one inherited from (3.4) for $p=d=1$, thanks 
to a sharper bound for $\mathrm{E}\left[\left|T(X)-D\left(\theta_{0}\right)\right|^{3}\right]$.

(2) The AR-bound is given by

$$
4.41456 \frac{\left\|h^{\prime}\right\|}{\sqrt{n}}+8 \frac{\|h\|}{n}+2 \frac{\left\|h^{\prime}\right\|}{\sqrt{n}}+80 \frac{\left\|h^{\prime}\right\|}{\sqrt{n}}\left(\frac{6}{n}+3\right)^{1 / 2},
$$

showing that our new bound is an improvement.

3.3.3. Empirical results. For a more complete picture, we also assess the accuracy of our results using simulation-based data. The process we follow is quite simple. We generate 10000 trials of $n=10,100,1000,10000$ and 100000 random i.i.d. observations from the exponential distribution $\operatorname{Exp}\left(\frac{1}{2}\right)$ (non-canonical case). As function $h$ we choose $h(x)=\frac{1}{x^{2}+2}$ with $h \in H,\|h\|=0.5$ and $\left\|h^{\prime}\right\|=\frac{3 \sqrt{1.5}}{16}$. With $\tilde{\mathrm{E}}[h(Z)]$ being the approximation of $\mathrm{E}[h(Z)]$ up to three decimal places, simple calculations yield $\tilde{\mathrm{E}}[h(Z)]=0.379$. Each trial gives an MLE $\hat{\theta}_{n}(\boldsymbol{X})$, hence we have $10000 \mathrm{em}-$ pirical values of $h\left(\sqrt{n i\left(\theta_{0}\right)}\left(\hat{\theta}_{n}(\boldsymbol{X})-\theta_{0}\right)\right)$ to compare to 0.379 . Taking the average provides a simulated estimation of $\left|\mathrm{E}\left[h\left(\sqrt{n i\left(\theta_{0}\right)}\left(\hat{\theta}_{n}(\boldsymbol{X})-\theta_{0}\right)\right)\right]-\mathrm{E}[h(Z)]\right|$, which we compare to the upper bound given in (3.11). Our bound provides a very strong improvement on the AR-bound (see Table 3.1). Of course, this estimated distance is only a lower bound to the true distance, as we have chosen a particular function $h$ instead of the supremum over all functions $h \in H$, but its calculation still provides an idea of the accuracy of our bounds. This closeness logically increases with the sample size and becomes quite sharp for $n \geq 100$.

TABLE 3.1. Simulation results for the $\operatorname{Exp}\left(\frac{1}{2}\right)$ distribution treated as a non-canonical exponential family

\begin{tabular}{r|r|r|r}
$n$ & $\mid \hat{\mathrm{E}}\left[h\left(\sqrt{n i\left(\theta_{0}\right)}\left(\hat{\theta}_{n}(\boldsymbol{X})-\theta_{0}\right)\right)\right]-\tilde{\mathrm{E}}[h(Z)]$ & New Bound & AR-bound \\
\hline \hline 10 & 0.0034 & 0.321 & 11.888 \\
\hline 100 & 0.0022 & 0.101 & 3.401 \\
\hline 1000 & 0.0012 & 0.032 & 1.058 \\
\hline 10000 & 0.0008 & 0.010 & 0.333 \\
\hline 100000 & 0.0004 & 0.003 & 0.105
\end{tabular}

\section{ACKNOWLEDGMENTS}

The authors would like to thank the Editor, an Associate Editor and an anonymous referee for helpful comments that led to an improvement of the paper. They further thank Gesine Reinert for various insightful comments and suggestions.

\section{References}

A. Anastasiou and G. Reinert. Bounds for the normal approximation of the maximum likelihood estimator. Bernoulli 23 (1), 191-218 (2017). MR3556771.

G. Casella and R. L. Berger. Statistical inference. The Wadsworth \& Brooks/Cole Statistics/Probability Series. Wadsworth \& Brooks/Cole Advanced Books \& Software, Pacific Grove, CA (1990). ISBN 0-534-11958-1. MR1051420. 
L. H. Y. Chen, L. Goldstein and Q.-M. Shao. Normal approximation by Stein's method. Probability and its Applications (New York). Springer, Heidelberg (2011). ISBN 978-3-642-15006-7. MR2732624.

D. R. Cox and E. J. Snell. A general definition of residuals. J. Roy. Statist. Soc. Ser. B 30, 248-275 (1968). MR0237052.

R. A. Fisher. Theory of Statistical Estimation. Mathematical Proceedings of the Cambridge Philosophical Society 22, 700-725 (1925). DOI: 10.1017/S0305004100009580.

C. J. Geyer. Asymptotics of maximum likelihood without the LLN or CLT or sample size going to infinity. In Advances in Modern Statistical Theory and Applications: A Festschrift in honor of Morris L. Eaton, pages 1-24. Eds.: G. Jones and X. Shen, Beachwood, OH: Institute of Mathematical Statistics (2013). DOI: 10.1214/12-IMSCOLL1001.

T. Mäkeläinen, K. Schmidt and G. P. H. Styan. On the existence and uniqueness of the maximum likelihood estimate of a vector-valued parameter in fixed-size samples. Ann. Statist. 9 (4), 758-767 (1981). MR619279.

I. Nourdin and G. Peccati. Normal approximations with Malliavin calculus, volume 192 of Cambridge Tracts in Mathematics. Cambridge University Press, Cambridge (2012). ISBN 978-1-107-01777-1. MR2962301.

G. Reinert. Couplings for normal approximations with Stein's method. In $M i$ crosurveys in discrete probability (Princeton, NJ, 1997), volume 41 of DIMACS Ser. Discrete Math. Theoret. Comput. Sci., pages 193-207. Amer. Math. Soc., Providence, RI (1998). MR1630415.

F. G. Tricomi and A. Erdélyi. The asymptotic expansion of a ratio of gamma functions. Pacific J. Math. 1, 133-142 (1951). MR0043948. 